\title{
Expectations of maser studies with FAST
}

\author{
Jiang Shui Zhang ${ }^{1}$, Di Li $^{2}$ and Jun Zhi Wang ${ }^{3}$ \\ ${ }^{1}$ Center for astrophysics, Guangzhou university, \\ Guangzhou 510006, \\ email: jszhang@gzhu.edu.cn \\ ${ }^{2}$ National Astronomical Observatories, Chinese Academy of Sciences \\ A20 Datun Road, Chaoyang District, Beijing 100012 \\ email: dili@nao.cas.cn \\ ${ }^{3}$ Department of Astronomy, Nanjing University, \\ 22 Hankou Road, Nanjing 210093, China \\ email: junzhiwang@nju.edu.cn
}

\begin{abstract}
The Five-hundred-meter Aperture Spherical radio Telescope (FAST) is being built by the Chinese and will be the largest single dish radio telescope in the world. FAST, with much increase in sensitivity, will give astronomers good opportunities to answer many fundamental questions in astronomy. Here we give a brief introduction of FAST and its enormous potential for studying Galactic and extragalactic masers.
\end{abstract}

Keywords. telescopes, masers

\section{Introduction}

The Five-hundred-meter Aperture Spherical radio Telescope (FAST) will be the largest single dish telescope in the world upon its finish in five years. Its location $\left(25^{\circ} .647222 \mathrm{~N}\right.$ and $\left.106^{\circ} .85583 \mathrm{E}\right)$ is about $170 \mathrm{~km}$ from Guiyang, the capital of Guizhou province in the southwest of China. Its construction began in March 2011 and its first light is expected at the end of 2016. With respect to existing radio telescopes, FAST has many unprecedented advantages: largest filled aperture, large sky coverage, extremely radio quiet site, etc. This will provide astronomers a good opportunity to explore many important science goals and the potential to obtain some big discoveries, such as, H I galaxy surveys, pulsar-black hole systems, extragalactic pulsars, radio signals from exoplanets (Nan et al. 2011).

One fundamental science proposal (Frontiers in radio astronomy and FAST Early Science) has been granted now, which will be carried out before the scheduled first light in 2016. The group of the proposal includes five science teams and one receiver group. The main goals of the proposal focus on defining key FAST programs and early science. In addition, tens of graduate students and young radio astronomers will be trained as part of the proposal.

FAST frequency coverage ranges from $70 \mathrm{MHz}$ to $3 \mathrm{GHz}$ and nine sets of receivers are planned according to the requirements of the science goals. For maser-related areas, $\mathrm{OH}$ masers $(\lambda \sim 18 \dot{\mathrm{cm}})$ could be the main object of FAST. Among those nine receivers, the L-wide single beam receiver (No. 7) can be used to detect both Galactic and extragalactic $\mathrm{OH}$ masers. The No. $3(0.28-0.56 \mathrm{GHz})$ and No. $4(0.56-1.02 \mathrm{GHz})$ receivers can be used to detect high redshift $\mathrm{OH}$ maser sources ( $\mathrm{OH}$ megamasers or gigamasers).

Here we present our investigation of existing $\mathrm{OH}$ maser observations and our proposed studies of both Galactic and extragalactic $\mathrm{OH}$ maser studies with the future FAST. 


\section{Investigations of $\mathrm{OH}$ maser studies}

Galactic OH masers: To date, more than $3000 \mathrm{OH}$ maser sources have been detected in our galaxy since their first discovery in interstellar space (Weaver et al. 1965). They can be basically classified as interstellar and circumstellar masers. Interstellar masers were considered to originate in dense molecular gas in star formation regions and circumstellar masers in the molecular circumstellar envelopes of evolved giant and supergiant stars (Reid 2002). About $80 \%$ of all Galactic OH masers belong to the latter (e.g., Lo 2005, Mu et al. 2010) and their catalogue and detailed information can be found in the Hamburg University database of circumstellar $\mathrm{OH}$ masers (http://www.hs.unihamburg.de/ st2b102/maserdb/index.html, also from Dr. Engels, these proceedings). In addition, $\mathrm{OH}$ masers with only $1720 \mathrm{MHz}$ emission are believed to be associated with supernova remnants (SNRs) and have proven to be excellent tracers of SNR-molecular cloud interactions. About $10 \%$ of SNRs $(\sim 20)$ were detected with this type of $\mathrm{OH}$ maser emission (e.g., Frail et al. 1996, Yusef-Zadeh et al. 1999, Hewitt et al. 2008, also Dr. Gray and Dr. Wardle, these proceeedings).

Searching for Galactic OH masers mainly consists of blind sky surveys and sampleselection surveys. Blind sky surveys were mostly performed long ago (in the 1970s and 1980s) with low sensitivity and the telescopes used include Parkes $64 \mathrm{~m}$, Onsala $25.6 \mathrm{~m}$, NRAO $42 \mathrm{~m}$, Effelsberg 100m etc. Many Galactic OH masers were found from these blind surveys (e.g., Caswell et al. 1980, Caswell \& Haynes 1983, Caswell \& Haynes 1987). Meanwhile, choosing different samples for searching for Galactic OH masers is also successful. Selected-samples mainly include color-selected IRAS samples (e.g., Eder et al. 1988, Lewis et al. 1990, te Lintel Hekkert 1991), star-forming-region samples (e.g., Szymczak et al. 2004, Wouterloot et al. 1993) and high mass protostellar object samples (e.g., Edris et al. 2007) etc.

Extragalactic $\mathbf{O H}$ masers: There are about $125 \mathrm{OH}$ megamasers so far $(\lambda \sim 18 \mathrm{~cm}$, Darling \& Giovanelli 2000, Chen et al. 2007, Fernandez et al. 2010, ten or so unpublished sources from Dr. Darling, also Dr. Willett, this symposium) since the first detection in galaxy IC4553 (Baan et al. 1982). The most luminous OH megamaser is IRAS14070+0525 with a redshift of 0.265 , detected by Arecibo (Baan et al. 1992). Host galaxies of those $125 \mathrm{OH}$ megamasers are (ultra)luminous infrared galaxies, which are believed to be the product of galaxy mergers (e.g., Clements et al. 1996). The rate of galaxy mergers was measured to be proportional to $(1+\mathrm{z})^{m}$ (m: 3 8, e.g, Le Fevre et al. 2000, Kim \& Sanders 1998). Therefore, we can expect more $\mathrm{OH}$ megamasers at high redshift, given that $\mathrm{OH}$ megamasers trace galaxy mergers.

Existing surveys of $\mathrm{OH}$ megamasers came from Arecibo $305 \mathrm{~m}$, NRAO $91 \mathrm{~m}$, JB MkIA $76 \mathrm{~m}$, Nancay $300 \mathrm{~m}$ and Parkes $64 \mathrm{~m}$ etc. The most successful survey so far is the upgraded Arecibo survey with a detection rate of $\sim 17 \%$ (Darling \& Giovanelli 2000). They chose a sample from the IRAS Point Source Catalog Redshift Survey $\left(f_{60}\right.$ um $>0.6$ Jy) (Saunders et al. 2000), with a declination range of $0^{\circ}-37^{\circ}$ (Arecibo sky coverage) and a redshift range of 0.1-0.3. Statistical results show a relation between OH and FIR luminosity of $L_{O H} \propto$ $L_{F I R}^{1.2 \pm 0.1}$ (Darling \& Giovanelli 2002a) and a luminosity function of $\phi \propto L_{O H}^{-0.6} \mathrm{Mpc}^{-3} \mathrm{dex}^{-1}$ (Darling \& Giovanelli 2002b, also Dr. Willett, this symposium).

\section{Expectations for maser studies}

Galactic OH masers: FAST has a sensitivity at least one order more than those telescopes used in the 1970s \& 1980s Galactic surveys. To achieve the same sensitivity limit, the FAST surveying speed would be 2 orders of magnitude better. This brings 
high efficiency for both sample selection surveys and Galactic plane blind sky surveys. As mentioned above, the L-wide single beam receiver could be used for these surveys. Certainly, an upgraded multi-beam receiver (L-wide receiver) will be better for wide area blind surveys. In addition, we believe that many more $1720 \mathrm{MHz} O \mathrm{OH}$ masers in SNRs could be detected with the high sensitivity FAST, which could help us understand the interaction between SNRs and adjacent molecular clouds, and further related sciences, such as the acceleration of relativistic particles, and the structure and physical conditions of the Galactic interstellar medium (Green et al. 1997).

Extragalactic $\mathbf{O H}$ masers: As discussed above, detections of $\mathrm{OH}$ megamasers come mainly from the Arecibo $305 \mathrm{~m}$ telescope. Compared with Arecibo, FAST has three times better raw sensitivity and about 10 times higher surveying speed. In addition, FAST has a zenith angle of 40 degrees, thanks to the deep karst depression and the innovative design of the active primary surface. It makes the declination range of FAST about -15 to 65 degrees (its latitude is $25^{\circ} .647222 \mathrm{~N}$ ), which is $2-3$ times Arecibo's sky coverage (Nan et al. 2011). Based on the FAST advantages, the following advances are expected:

1) Numbers of $\mathrm{OH}$ megamasers: three times better sensitivity can extend the detection limit by a factor of $\sim \sqrt{3}$ in terms of distance, thus increasing the sample size of detectable sources by a factor of $\sim 3 \sqrt{3}$ times. Taking into account its $2-3$ times sky coverage increase, we can expect the number of $\mathrm{OH}$ megamasers to increase 10-20 times, assuming a similar detection rate and luminosity function. Thus more than $1000 \mathrm{OH}$ megamasers could be detected by FAST.

2) Higher sensitivity and larger sky coverage provide more opportunities to detect high redshift $\mathrm{OH}$ megamasers or Gigamasers. The Arecibo survey gives a typical rms flux density of $0.65 \mathrm{mJy}$ or so for a 12 minute integration. Given FAST with 3 times better sensitivity, the rms value of a FAST similar survey is $\sim 0.22 \mathrm{mJy}$ for the same integration time. According to a plot of sensitivity thresholds of $\mathrm{OH}$ megamaser detections (Darling \& Giovanelli 2002b), FAST could detect most $\mathrm{OH}$ megamsers with $L_{O H}>10^{3} L_{\odot}$ out to $\mathrm{z} \sim 1$ and could detect $\mathrm{OH}$ gigamasers out to roughly $\mathrm{z} \sim 2$ in a 12 minute integration.

3) The most distant $\mathrm{OH}$ megamaser $(1720 \mathrm{MHz})$ was detected by the Green Bank Telescope in a gravitationally lensed source PMN J0134-0931 (Kanekar et al. 2005). FAST detections of $\mathrm{OH}$ maser emission could be expected in similar, but more distant (e.g., $\mathrm{z}>1$ ) sources.

\section{Acknowledgements}

This work is supported by the China Ministry of Science and Technology under the State Key Development Program for Basic Research (2012CB821800) and the Natural Science Foundation of China (No. 11043012, 11178009). We made use of the NASA Astrophysics Data System Bibliographic Services (ADS) and the NASA/IPAC extragalactic Database (NED), which is operated by the Jet Propulsion Laboratory, California Institute of Technology, under contract with NASA.

\section{References}

Baan, W. A., Wood, P. A. D., \& Haschick, A. D. 1982, ApJ, 260, L49

Baan, W. A., Rhoads, J., Fisher, K., Altschuler, D. R., \& Haschick, A. D. 1992, ApJ, 396, L99

Caswell, J. L., Haynes, R. F., \& Goss, W. M. 1980, Australian Journal of Physics, 33, 639

Caswell, J. L. \& Haynes, R. F. 1983, Australian Journal of Physics, 36, 361

Caswell, J. L. \& Haynes, R. F. 1987, Australian Journal of Physics, 40, 215

Chen, P. S., Shan, H. G., \& Gao, Y. F. 2007, AJ, 133, 496 
Clements, D. L., Sutherland, W. J., McMahon, R. G., \& Saunders, W. 1996, MNRAS, 279, 477

Darling, J. \& Giovanelli, R. 2000, AJ, 119, 3003

Darling, J. \& Giovanelli, R. 2002a, AJ, 124, 100

Darling, J. \& Giovanelli, R. 2002b, ApJ, 572, 810

Eder, J., Lewis, B. M., \& Terzian, Y. 1988, ApJS, 66, 183

Edris, K. A., Fuller, G. A., \& Cohen, R. J. 2007, A\& A, 465, 865

Fernandez, M. X., Momjian, E., Salter, C. J., \& Ghosh, T. 2010, AJ, 139, 2066

Frail, D. A., Goss, W. M., Reynoso, E. M., Giacani, E. B., Green, A. J., \& Otrupcek, R. 1996, $A J, 111,1651$

Green, A. J., Frail, D. A., Goss, W. M., \& Otrupcek, R. 1997, AJ, 114, 205

Hewitt, J. W., Yusef-Zadeh, F., \& Wardle, M. 2008, ApJ, 683, 189

Kanekar, N., Carilli, C. L., \& Langston, G. I. et al. 2005, PhRvL, 95, 1301

Kim, D.-C. \& Sanders, D. B. 1998, ApJS, 119, 41

Le Fvre, O., Abraham, R., \& Lilly, S. J. 2000, MNRAS, 311, L565

Lewis, B. M., Eder, J., \& Terzian, Y. 1990, ApJ, 362, 634

Lo, K. Y. 2005, ARA\&A, 43, 625

Mu, J. M., Esimbek, J., Zhou, J. J., \& Zhang, H. J. 2010, RAA, 2, 166

Nan, R. D., Li, D., \& Jin, C. J. et al. 2011, IJMPD, 20, 989

Reid, M. 2002, IAU Symp., 206, 506

Saunders, W., Sutherland, W. J., \& Maddox, S. J. et al. 2000, MNRAS, 317, 55

Szymczak, M. \& Grard, E. 2004, A\& A, 423, 209

te Lintel Hekkert, P. 1991, A\&GA, 248, 209

Weaver, H., Williams, D. R. W., Dieter, N. H., \& Lum, W. T. 1965, Nature, 208, 29

Wouterloot, J. G. A., Brand, J., \& Fiegle, K. 1993, A\&SAS, 98, 589

Yusef-Zadeh, F., Goss, W. M., Roberts, D. A., Robinson, B., \& Frail, D. A. 1999, ApJ, 527, 172 